\title{
Verification and Comparison of Transition Systems
}

\author{
André Arnold \\ LaBRI * \\ Université Bordeaux I
}

\begin{abstract}
The notion of a transition system is of fundamental importance for studying the semantics of systems of interacting processes. Firstly, this notion is convenient for modeling these systems. In particular, the synchronized product of transition systems allows us to describe a system from the description, by transition systems, of its components, and from a description of the interactions between these components by giving the actions or events that may simultaneously occur in the system. Secondly, the notion of a transition system is the basis on which one can develop semantic notions such as verification of properties, by designing logics having transition systems as models, or comparison of transition systems, by structural equivalences defined by homomorphisms or logical equivalences related to logics for transition systems.

In this paper we present a short survey of these basic notions.
\end{abstract}

\section{Introduction}

Most of the works on the semantics of "concurrent", or "communicating", or "interacting" processes, rely on the notion of an automaton. This notion allows us not only to model more or less precisely the behaviour of a system, but to develop tools for verifying the modeled system: from its representation as an automaton, it is easy to observe and to verify some of its properties, like deadlocks, or to compare it with some others.

Although automata theory was mainly developed in relation to formal language theory and its application to lexical and syntactic analysis, this theory was also used in other domains, in particular in the semantics of sequential while-programs $[22,30]$. The well-known flowcharts are finite automata describing the control flow of programs. More generally, a finite automaton, consisting of states and labeled transitions, allows us to describe a system whose state evolves over time. Indeed, automata were introduced for this reason, as pointed out by Minsky [27], since they originate with the modeling of neurons, and the celebrated Kleene's theorem

\footnotetext{
*Research Unit of the Centre National de la Recherche Scientifique nº 1304
} 
characterizing languages recognized by a finite automaton refer to behaviours of nerve nets.

In the case of a flowchart the state of a system is the program counter, but the modeling of a sequential program can be enriched by considering the state consisting not only in the program counter but also in the values of some (or all) of its variables (as long as they have a finite range; otherwise the associated automation will obviously be no longer finite); each action possibly performed by the program is actually performed only when the program is in some particular states, and this action implies a change of state; thus, actions are represented by transitions of an automaton.

This way of representing, by an automaton, a system whose state evolves because of some actions or because of the occurrences of some events applies to many situations, among which systems of processes appear quite naturally. For systems of processes interacting by shared variables, Karp and Miller [23] have extended the model of sequential processes proposed by lanov [22] by taking into account the fact that some actions performed by the processes of the system can modify disjoint parts of the common storage. The control of the system remains centralized; it is still represented by a finite automaton. But the actions which test or modify the content of the storage test or modify only a part of this storage. Adding the states of the storage to the states of the control, one gets a description of the whole system by a finite automaton, provided, of course that the storage can contain only a finite number of different values. If the control is itself distributed, that is to say if the program counter is, like the storage, a vector, and if action modifies only some components of this vector, the automaton modeling such a system belongs to a remarkable class of automata defined and studied by Zielonka [32], the so-called asynchronous automata.

Other models of systems of processes have been proposed; among them are Petri nets [7], and process calculi à la Milner, like CCS [25, 26, 19] and Meije [6]. Without going into the details, one can admit that all these models amount to specifying sets of states and of transitions between these states. For instance, the states of a Petri net are its markings and transitions are realized by firing one or several transitions of the net. In process calculi, states are terms of a process algebra and transitions are defined by the operational semantics of the calculus which explains how a term is rewritten into another one.

One can therefore consider that the fundamental notion for formally describing a system of processes is the one of an automaton. To emphasize the fact that these automata are not devices used to recognize languages, but that they are formalizations of a structure consisting of states and transitions, we call them transition systems. In contrast with transilion systems introduced by Keller [24] to model systems of processes, the transitions of transition systems we are using are labeled by action or event names, although it is not absolutely necessary: as we shall see it later on, what we need is to distinguish some particular sets of transitions, for instance the set of all transitions caused by a given event. Also, while the definition of a classical automaton includes one or several initial states and a set of final states, 
the definition of a transition system does not imply the existence of such states, or, more precisely, it is possible to distinguish any number of sets of states according to the need of the modeling. For instance, when modeling a CSP program [21] by a transition system, we need to define the set of success states corresponding to a normal termination of the program, and the set of failure states.

In the first section of this paper, we introduce transition systems and we explain how they allow us to express the behaviour of processes. We also explain how to model the interactions between the processes of a system by explicitely giving the sets of vectors of actions or events that can occur simultaneously in the system. The importance of this operation comes from the fact that all the communication and synchronization constraints can be expressed this way. Then, we define a fundamental operation on transition systems, the synchronized product. This operation, introduced by Arnold and Nivat [28, 5, 2], is used to construct a transition system modeling a system of concurrent processes from the transition systems modeling each component process and from a description of the interactions between these components.

To express properties of a transition system, one needs a formal language, called a logic (although this name is somewhat abusive, since we are not interested in any notion of a proof in these logics). In the second section, we introduce some of these logics, and we show how it is possible to verify the properties stated in these logics.

One of the main consequences of the existence of a formal definition of the semantics of a system of processes is that it is a basis for a precise definition of the equivalence of systems. It becomes possibles to compare two systems directly or to check that an implementation of a system obeys its specification when both can be expressed in the same formalism, namely as transition systems. In the third section, we introduce two kinds of equivalences: structural equivalences defined via transition system homomorphisms, and logical equivalences defined in relation with the satisfiability of logical formulas. Then we show how these two kinds of equivalences can be related.

\section{Modeling interacting systems of processes}

\subsection{Modeling processes}

\subsubsection{Labeled transition systems}

A labcled transition system over an alphabet $A$ of actions or events is a tuple $\mathcal{A}=$ $\langle S, T, \alpha, \beta, \lambda\rangle$ where

- $S$ is a finite set of states,

- $T$ is a finite set of transitions,

- $\alpha, \beta: T \longrightarrow S$ are the mappings which associate with every transition $t$ its source state $\alpha(t)$ and its target state $\beta(t)$,

- $\lambda: T \longrightarrow A$ labels a transition $t$ by the action or event $\lambda(t)$ which causes this transition. 
We assume that there never exist two different transitions with the same label between the same two states, i.e. the mapping $\langle\alpha, \lambda, \beta\rangle: T \longrightarrow S \times A \times S$ is injective.

\subsubsection{Parameterized transition systems}

A parameterized transition system is a labeled transition system given with some sets of designed states and some sets of designed transitions, called parameters. The role of these parameters is to give some additional informations on the transition system; it is the case when some states play a special role or when some transitions play a special role which is not specified by the label of the transition. Some example of such situations will be given below.

Example 1. A boolean variable Let us consider a boolean variable. It has two states, denoted by 0 and 1 , according to the current value ( 0 or 1 ) of the variable. The set $A$ of actions performed by such a boolean variable contains

to0 which means that the variable is set to 0 ,

to1 which means that the variable is set to 1 ,

is 0 which tests whether the value of the variable is 0 ,

is 1 which tests whether the value of the variable is 1 ,

e which does nothing.

The first two actions modify the value of the variable, i.e. its state, in an obvious way. The two tests can be executed only if the variable has the tested value, and this value is not modified. The last action, when executed, does not change the value of the variable. As we shall see later on (example 3), this null action is a way to express the possibility of occurrence of events which does not modify the state of the variable. Therefore the transition system has eight transitions: for each one of these transitions we give, in the following table, its source state, its label, and its target state.

$$
\begin{aligned}
& t_{1}: 0 \mapsto \mathrm{e} \rightarrow \mathbf{0} \\
& t_{2}: 0 \mapsto \text { to0 } \rightarrow 0 \\
& t_{3}: 0 \mapsto \text { to } \rightarrow 1 \\
& t_{4}: 0 \mapsto \text { is } \rightarrow 0 \\
& t_{5}: 1 \mapsto \mathrm{e} \rightarrow 1 \\
& t_{6}: 1 \mapsto \text { to0 } \rightarrow 0 \\
& t_{7}: 1 \mapsto \text { to } \rightarrow 1 \\
& t_{8}: 1 \mapsto \text { is } 1 \rightarrow 1
\end{aligned}
$$

Example 2. A sequential program Let us consider the program fragment 


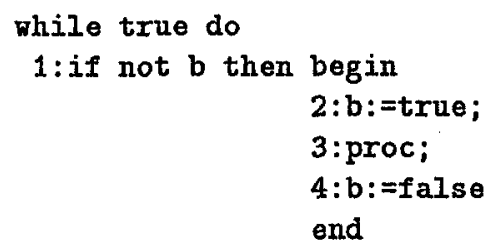

With this program, we associate a transition system $\mathcal{P}$ having four states: 12 , 3 et 4 (the values of "program counter"). Actions performed by $\mathcal{P}$ are $b:=$ false and $b:=$ true, proc, and the two tests, denoted $b y=$ true? and $b=$ false? which correspond to the cases where the tested value of $b$ has been found respectively equal to true and false. Thus we get the following transitions

$$
\begin{array}{cccc}
t_{1}^{\prime}: & 1 & \mapsto b=\text { true? } \rightarrow & 1 \\
t_{2}^{\prime}: & 1 & \mapsto b=\text { false? } \rightarrow & 2 \\
t_{3}^{\prime}: & 2 & \mapsto b:=\text { true } \rightarrow & 3 \\
t_{4}^{\prime}: & : & \mapsto \text { proc } \rightarrow & 4 \\
t_{5}^{\prime}: & 4 & \mapsto b:=\text { false } \rightarrow & 1
\end{array}
$$

Each component of a system of processes as well the processes themselves as some other ressources such as shared variables, communication channels, etc., can be modelized by transition systems.

\subsection{Modeling interactions}

Before explaining how to model the interactions between the components of a system of processes, we show how one can obtain the transition system modeling the whole system, when there is no interaction at all.

\subsubsection{Free products of transition systems.}

Let us consider $n$ transition systems $\mathcal{A}_{i}=\left\langle S_{i}, T_{i}, \alpha_{i}, \beta_{i}, \lambda_{i}\right\rangle$ labeled by $A_{i}$, for $i=$ $1, \ldots, n$. The free product $\mathcal{A}_{1} \times \cdots \times \mathcal{A}_{n}$ of these $n$ transition systems is the transition system $\mathcal{A}=\langle S, T, \alpha, \beta, \lambda\rangle$ labeled by $A_{1} \times \cdots \times A_{n}$, defined by

$$
\begin{aligned}
S & =S_{1} \times \ldots \times S_{n}, \\
T & =T_{1} \times \ldots \times T_{n}, \\
\alpha\left(t_{1}, \ldots, t_{n}\right) & =\left\langle\alpha_{1}\left(t_{1}\right), \ldots, \alpha_{n}\left(t_{n}\right)\right\rangle, \\
\beta\left(t_{1}, \ldots, t_{n}\right) & =\left\langle\beta_{1}\left(t_{1}\right), \ldots, \beta_{n}\left(t_{n}\right)\right\rangle, \\
\lambda\left(t_{1}, \ldots, t_{n}\right) & =\left\langle\lambda_{1}\left(t_{1}\right), \ldots, \lambda_{n}\left(t_{n}\right)\right\rangle .
\end{aligned}
$$

If the transition system $\mathcal{A}$ is in the global state $s=\left\langle s_{1}, \ldots, s_{n}\right\rangle$, each component transition system, $\mathcal{A}_{i}$, is in the state $s_{i}$; it can perform, independently of every other component, a transition $t_{i}$ which will set it in the state $s_{i}^{\prime}$. Then, the transition system $\mathcal{A}$ is in the global state $s^{\prime}=\left\langle s_{1}^{\prime}, \ldots, s_{n}^{\prime}\right\rangle$ after having performed the global transition $t=\left\langle t_{1}, \ldots, t_{n}\right\rangle$, corresponding to the global action $\lambda(t)$. 
Such a definition makes sense if we assume that in a system all components simultaneously execute a transition, i.e., there is a sequence of time slices such that in each slice, each component executes one and only one transition.

\subsubsection{Synchronization vectors}

When processes interact, it is no longer true that any global action can occur in the system. Indeed, interactions can be described by giving those global actions that may occur in the system and those that may not.

Let us consider the transition system $\mathcal{B}$ of Example 1 representing a boolean variable and the transition system $\mathcal{P}$ of Example 2 representing a program which uses this variable.

Since $\mathcal{B}$ has two states and eight transitions and $\mathcal{P}$ has four states and five transitions, their free product has eight states and forty transitions and contains, for instance, the global transition consisting of $t_{2}$ of $\mathcal{B}$ and $t_{3}^{\prime}$ of $\mathcal{P}$ from the global state $\langle 0,2\rangle$ to $\langle 0,3\rangle$ labeled by the global action $\langle$ to $0, b:=$ true $\rangle$.

But the intuitive meaning of $b:=$ true, executed by $p$, is to set the boolean variable to 1 . This variable must simultaneously execute a transition labeled by to1. Conversely, if the variable executes to 1 , it is being set to 1 by $\mathcal{P}$, which executes $b:=$ true. In other words, (to1, $b:=$ true) is the only global action whose first component is to 1 ; it is also the only one whose second component is $b:=$ true. In the same way, if $\mathcal{P}$ executes $b=$ false? which means that the value of the variable has been found equal to 0 , the variable must simultaneously execute an action returning this value, that is is0, and the only global action having is0 as first component, or $b=$ false? as second component, is 〈 is $0, b=$ false? $\rangle$.

Finally, $\mathcal{P}$ does not interact with the variable when executing proc; thus the variable does nothing, i.e., executes e.

Thus, allowed global actions are:

$$
\begin{aligned}
& \text { (to1, b:=true) } \\
& \langle\text { to0,b:=false } \\
& \text { (is1, } b=\text { false?) } \\
& \text { 〈is0, } b=\text { true? } \\
& \text { (e, proc) }
\end{aligned}
$$

Now, we formally define interactions between components of a system. If $A_{1}, \ldots, A_{n}$ are alphabets of actions or events, a synchronization consiraint is a subset of the cartesian product $A_{1} \times \cdots \times A_{n}$. Each member of this subset is a synchronization vector and represents an allowed global action of the system.

\subsection{Synchronized products of transition systems}

If $\mathcal{A}_{i}, i=1, \ldots, n$ are transition systems respectively labeled by the alphabets $A_{i}$, and if $I \subseteq A_{1} \times \cdots \times A_{n}$ is a synchronization constraint, the synchronized product 
of the $\mathcal{A}_{i}$ with respect to $I$, is the sub-transition system of the free product of the $\mathcal{A}_{i}$, containing only the global transitions labeled by a member of $I$.

Therefore, a system of processes is also modeled by a transition system.

Example 3. The synchronized product of $\mathcal{B}$ and $\mathcal{P}$ with respect to the synchronization constraint given above is

$$
\begin{aligned}
& \langle 1,1\rangle \mapsto\langle\text { is } 1, b=\operatorname{true} ?\rangle \rightarrow\langle 1,1\rangle \\
& \langle 1,2\rangle \mapsto\langle\text { to } 1, b:=\text { true }\rangle \rightarrow\langle 1,3\rangle \\
& \langle 1,3\rangle \quad \mapsto\langle e, \text { proc }\rangle \rightarrow \quad\langle 1,4\rangle \\
& \langle 1,4\rangle \mapsto\langle\text { too }, \mathrm{b}:=\text { false }\rangle \rightarrow\langle 0,1\rangle \\
& \langle 0,1\rangle \mapsto\langle\text { iso }, b=\text { false? }\rangle \rightarrow\langle 0,2\rangle \\
& \langle 0,2\rangle \mapsto\langle\text { to1 }, \mathrm{b}:=\text { true }\rangle \rightarrow\langle 1,3\rangle \\
& \langle 0,3\rangle \quad \mapsto\langle e, \text { proc }\rangle \rightarrow \quad\langle 0,4\rangle \\
& \langle 0,4\rangle \mapsto\langle\text { too }, \mathrm{b}:=\text { false }\rangle \rightarrow\langle 0,1\rangle
\end{aligned}
$$

\section{Logics for transition systems}

In order to check the correctness of a system of processes described by a transition system, one has to explicitly express the properties it must have: without such informations the answer to the question "is this system correct?" is always "yes".

Thus, checking correctness of a system requires

- a language to express properties, and

- a definition of whether or not a given transition system has a given property.

This is typically what logics are made for: properties are expressed by formulas, and the satisfaction relation correspond to the second point. On the other hand, we need not any proof system, so that the term of "logic" is used in a wider sense than usual.

\subsection{Propositional logics}

Let us start with two classical examples of propositional logics for transition systems.

\subsubsection{Hennessy-Milner logic}

The formulas of the Hennessy-Milner logic [20] are built up from

- the constants 1 et 0 (true and false), and the usual logical operators $\vee, \wedge, \neg$, - a unary operator $\langle a\rangle$, for each letter $a$ of the alphabet $A$.

Let $\mathcal{A}=\langle S, T, \alpha, \beta, \lambda\rangle$ be a given transition system and $F$ be a given formula. For any state $s$ of $S$ we define the satisfaction relation $\mathcal{A}, s \vDash F$ by induction on the construction of $F$ : 
- $\mathcal{A}, s \vDash 1, \mathcal{A}, s \not \neq 0$,

- $\mathcal{A}, s \vDash F \vee F^{\prime}$ iff $\mathcal{A}, s \models F$ or $\mathcal{A}, s \models F^{\prime}$,

- $\mathcal{A}, s \vDash F \wedge F^{\prime}$ iff $\mathcal{A}, s \vDash F$ and $\mathcal{A}, s \vDash F^{\prime}$,

- $\mathcal{A}, s \vDash \neg F$ iff $\mathcal{A}, s \not k F$,

- $\mathcal{A}, s \vDash\langle a\rangle F$ iff there is a transition $s \mapsto a \rightarrow s^{\prime} \in T$ such that $\mathcal{A}, s^{\prime} \vDash F$.

\subsubsection{CTL*}

$\mathrm{CTL}^{*}[15,14]$ is a logic with two kinds of formulas: state formulas and path formulas, and does not take the labels of transitions into account. On the other hand it uses a given set of propositional symbols.

A path in a transition system $\mathcal{A}=\langle S, T, \alpha, \beta, \lambda\rangle$ is a finite or infinite sequence $p=t_{1}, t_{2}, \ldots$, of transitions such that for all $i, \beta\left(t_{i}\right)=\alpha\left(t_{i+1}\right)$. The source of this path, denoted by $\alpha(p)$, is $\alpha\left(t_{1}\right)$.

Formulas are built up according to the following rules: a state formula is

- a propositional symbol, or the constants 1 or 0 ,

- $F \vee F^{\prime}, F \wedge F^{\prime}$, and $\neg F$, where $F$ and $F^{\prime}$ are state formulas,

- $E G$ where $G$ is a path formula;

a path formula is

- the constants 1 or 0 ,

- $G \vee G^{\prime}, G \wedge G^{\prime}$, and $\neg G$, where $G$ and $G^{\prime}$ are path formulas,

- NG and $G U G^{\prime}$, where $G$ and $G^{\prime}$ are path formulas,

- $B F$, where $F$ is a state formula.

Let us consider a transition system $\mathcal{A}$ and subset $P_{\mathcal{A}}$ of $S$ for any propositional symbol $P$. The satisfaction relation is defined by

- $\mathcal{A}, s \vDash P$ iff $s \in P_{\mathcal{A}}, \mathcal{A}, s \vDash 1, \mathcal{A}, s \not \neq 0$,

- $\mathcal{A}, s \vDash F \vee F^{\prime}$ iff $\mathcal{A}, s \vDash F$ or $\mathcal{A}, s \models F^{\prime}$,

- $\mathcal{A}, s \vDash F \wedge F^{\prime}$ iff $\mathcal{A}, s \models F$ and $\mathcal{A}, s \models F^{\prime}$,

- $\mathcal{A}, s \vDash \neg F$ iff $\mathcal{A}, s \nvdash F$,

- $\mathcal{A}, s \vDash E G$ iff there is an infinite path $p$ of source $s$ such that $\mathcal{A}, p \vDash G$,

- $\mathcal{A}, p \models 1, \mathcal{A}, p \nvdash \mathbf{0}$,

- $\mathcal{A}, p \vDash G \vee G^{\prime}$ iff $\mathcal{A}, p \vDash G$ or $\mathcal{A}, p \vDash G^{\prime}$,

- $\mathcal{A}, p \models G \wedge G^{\prime}$ iff $\mathcal{A}, p \vDash G$ and $\mathcal{A}, p \models G^{\prime}$,

- $\mathcal{A}, p \models \neg G$ iff $\mathcal{A}, p \forall G$,

- $\mathcal{A}, p \models N G$ iff $p=t \cdot p^{\prime}$ and $\mathcal{A}, p^{\prime} \vDash G$,

- $\mathcal{A}, p \vDash G U G^{\prime}$ iff

- $\mathcal{A}, p \models G^{\prime}$ or

- $p=t_{1} t_{2} \cdots t_{n} \cdot p^{\prime}$ with $\mathcal{A}, p^{\prime} \vDash G^{\prime}$ and $\forall i \in\{1, \ldots, n\}, \quad \mathcal{A}, l_{i} \cdots t_{n} \cdot p^{\prime} \vDash G$,

- $\mathcal{A}, p \vDash B F$ iff $\mathcal{A}, \alpha(p) \vDash F$. 


\subsubsection{Propositional logics as free algebras}

These two logics, and many others, (see [13] for a survey) fall in the scope of the following definition.

Let $\mathcal{R}$ be a set of sorts and $\Omega$ be a set of sorted operators, i.e., each operator $\omega$ has an arity $\delta(\omega)$ and a sort $\rho_{1} \cdots \rho_{\delta(\omega)} \rightarrow \rho$. A formula is a well-formed ground term over $\Omega$.

An $\Omega$-poweralgebra $\mathcal{A}$ is a family $\left(E_{\rho}\right)_{\rho \in \mathcal{R}}$ of sets together with a mapping $\omega_{\mathcal{A}}: \mathcal{P}\left(E_{\rho_{1}}\right) \times \cdots \times \mathcal{P}\left(E_{\rho_{n}}\right) \rightarrow \mathcal{P}\left(E_{\rho}\right)$ for any $\omega \in \Omega$ of sort $\rho_{1} \cdots \rho_{n} \rightarrow \rho$.

Therefore, each formula $F$ of sort $\rho$ has an interpretation $F_{\mathcal{A}} \subseteq E_{\rho}$ and the satisfaction relation is defined by $\mathcal{A}, r \models F$ iff $r \in F_{\mathcal{A}}$.

For instance, Hennessy-Milner logic can be defined by the union of the two set of operators $\{\mathbf{1}, \mathbf{0}, \vee, \wedge, \neg\}$ and $\{\langle a\rangle \mid a \in A\}$, with only one sort. The interpretation of logical operators in a transition system $\mathcal{A}$ are set-theoretical operations on $\mathcal{P}(\mathcal{S})$, while the interpretation of $\langle a\rangle$ is defined by

$$
\langle a\rangle_{\mathcal{A}}(X)=\left\{s \in S \mid \exists s^{\prime} \in X, \exists t \in T: t=s \mapsto a \rightarrow s^{\prime}\right\} .
$$

The logic CTL ${ }^{*}$ can be defined in the same way, it has two sorts: states and paths, so that to be quite rigorous, it must contain two sets of logical operators, one for each sort, but the sort of such an operator can be determined from its context. As another example, we introduce the logic proposed by Dicky [12]. It has two sorts, states and transitions, denoted by $\sigma$ and $\tau$. it contains usual logical operators and constants, of both sorts, and the specific unary operators $s r c$ and $t g t$ of sort $\tau \rightarrow \sigma$, and in and out of sort $\sigma \rightarrow \tau$. Their interpretation in a transition system $\mathcal{A}$ is defined by

$$
\begin{aligned}
& \text { - } \operatorname{src}_{\mathcal{A}}(R)=\{\alpha(t) \mid t \in R\}, \quad \operatorname{tgt}_{\mathcal{A}}(R)=\{\beta(t) \mid t \in R\}, \\
& \text { - } \operatorname{in}_{\mathcal{A}}(Q)=\{t \in T \mid \beta(t) \in Q\}, \quad \operatorname{out}_{\mathcal{A}}(Q)=\{t \in T \mid \alpha(t) \in Q\} .
\end{aligned}
$$

In this algebra, we can generalize the operator $\langle a\rangle$ of Hennessy-Milner logic: we define the new binary operator $\left\langle Y_{\tau}\right\rangle X_{\sigma}$, of sort $\tau \sigma \rightarrow \sigma$, by $\operatorname{src}\left(Y_{\tau} \wedge \operatorname{in}\left(X_{\sigma}\right)\right.$. In a transition system $\mathcal{A},\langle R\rangle Q$ is interpreted as the set

$$
\left\{s \in S \mid \exists a \in A, \exists t=s \mapsto a \rightarrow s^{\prime} \in R: s^{\prime} \in Q\right\},
$$

and if $R$ is the set of all transitions labeled by $a$, this is obviously equal to the interpretation of $\langle a\rangle$.

\section{$2.2 \mu$-calculi}

Let us consider the state formula $F=E\left(B P U B P^{\prime}\right)$ of $\mathrm{CTL}^{*}$ and its interpretation $F_{\mathcal{A}}$ in a transition system $\mathcal{A}$ where $P$ and $P^{\prime}$ are respectively interpreted as $P_{\mathcal{A}}$ and $P_{\mathcal{A}}^{\prime}$. It can be easily shown that $F_{\mathcal{A}}$ is the least fixpoint for inclusion of the equation $X=P_{\mathcal{A}}^{\prime} \cup\left(P \cap N_{\mathcal{A}} X\right)$.

More generally, let us consider an $\Omega$-term $t\left(X_{0}, X_{1}, \ldots, X_{n}\right)$ of sort $\rho_{0}$ over the set $\left\{X_{0}, X_{1}, \ldots, X_{n}\right\}$ of variables, where $X_{i}$ is of sort $\rho_{i}$. Let us assume that for any $\Omega$-poweralgebra $\mathcal{A}$, the mapping $t_{\mathcal{A}}: \mathcal{P}\left(E_{\rho_{0}}\right) \times \mathcal{P}\left(E_{\rho_{1}}\right) \times \cdots \times \mathcal{P}\left(E_{\rho_{n}}\right) \rightarrow \mathcal{P}\left(E_{\rho_{0}}\right)$ is 
monotonic for inclusion with respect to all its argument (for the logics considered above, there is a syntactically defined set of terms having this property). Then there exists two monotonic mappings

$t_{\mathcal{A}}^{*}: \mathcal{P}\left(E_{\rho_{1}}\right) \times \cdots \times \mathcal{P}\left(E_{\rho_{n}}\right) \rightarrow \mathcal{P}\left(E_{\rho_{0}}\right)$ and $t_{\mathcal{A}}^{\infty}: \mathcal{P}\left(E_{\rho_{1}}\right) \times \cdots \times \mathcal{P}\left(E_{\rho_{n}}\right) \rightarrow \mathcal{P}\left(E_{\rho_{0}}\right)$ such that $t_{\mathcal{A}}^{*}\left(P_{1}, \ldots, P_{n}\right)$ (resp. $\left.t_{\mathcal{A}}^{\infty}\left(P_{1}, \ldots, P_{n}\right)\right)$ is the least (resp. greatest) fixpoint of $X=t_{\mathcal{A}}\left(X, P_{1}, \ldots, P_{n}\right)$. Thus we form new formulas by prefixing terms by $\mu X$ or $\nu X$ where the interpretation of $\mu X . t\left(X, X_{1}, \ldots, X_{n}\right)$ (resp. $\left.\nu X . t\left(X, X_{1}, \ldots, X_{n}\right)\right)$ in $\mathcal{A}$ is $t_{\mathcal{A}}^{*}\left(X_{1}, \ldots, X_{n}\right)$ (resp. $\left.t_{\mathcal{A}}^{\infty}\left(X_{1}, \ldots, X_{n}\right)\right)$.

For instance, the formula $E\left(B P \cup B P^{\prime}\right)$ can be rewritten as $\mu X . P^{\prime} \vee(P \wedge N X)$.

By iterating this process, one can define a whole hierarchy of new operators.

Example 4. Let us define the following operators

$$
\begin{aligned}
\operatorname{Pred}(X) & =\operatorname{src}(\operatorname{in}(X)), \\
\operatorname{Coreach}(X) & =\mu Y . \operatorname{Pred}(X \vee Y), \\
\operatorname{Live}(X) & =\nu Y \cdot \operatorname{Coreach}(X \wedge Y) .
\end{aligned}
$$

The interpretation of $\operatorname{Live}(X)$ in $\mathcal{A}$ is the set of sources of paths going through the interpretation of $X$ infinitely often.

\subsection{Verification of formulas}

Now, the problem of verification of a transition system $\mathcal{A}$ amounts to computing the interpretation in $\mathcal{A}$ of formulas of a given logic. To do that it is sufficient to be able to compute $\omega_{\mathcal{A}}\left(P_{1}, \ldots, P_{n}\right)$ when the sets $P_{1}, \ldots, P_{n}$ are given. For most of the usual logics for transition systems, this can be done in time linear in the the size of the transition system $\mathcal{A}$ (cf. [8] for instance). Moreover, it has been shown in [3] that computing $\mu X . t\left(X, X_{1}, \ldots, X_{n}\right)$ in $\mathcal{A}$ can be also be done in lime linear in the size of $\mathcal{A}$ for terms $t$ of the Dicky's logic; this algorithm, which is quadratic in the size of $t$, has been improved independently in [9], [1], and [31], where it is also linear in the size of $t$.

\section{Comparison of transition systems}

When comparing transition systems, an important point to consider is in which respect they may be considered to be equivalent. Very roughly speaking one can say that two transition systems are equivalent if they share common characteristic features. Then the point is to define what a common characteristic feature is. We propose two ways of defining such a notion. The first one is based upon the very "structure" of a transition system, characterized by homomorphisms; the second one is based upon the logical properties of a transition system. Then we show that in some cases these two definitions can be related. 


\subsection{Structural equivalences}

As it is usual in mathematics, one can consider that the characteristic features of a transition system are those that are preserved under morphisms of a given family. The image of a transition system under such a morphism is called its quotient and two objects are equivalent if they have a common quotient. This leads us to the following definitions.

Let $\mathcal{A}=\langle S, T, \alpha, \beta, \lambda\rangle$ and $\mathcal{A}^{\prime}=\left\langle S^{\prime}, T^{\prime}, \alpha^{\prime}, \beta^{\prime}, \lambda^{\prime}\right\rangle$ be two labeled transition systems. A homomorphism $h$ from $\mathcal{A}$ into $\mathcal{A}^{\prime}$ is a pair of mappings ( $h_{\sigma}: S \rightarrow S^{\prime}$, $\left.h_{\tau}: T \rightarrow T^{\prime}\right)$ such that $\forall t \in T, \alpha^{\prime}\left(h_{\tau}(t)\right)=h_{\sigma}(\alpha(t)), \beta^{\prime}\left(h_{\tau}(t)\right)=h_{\sigma}(\beta(t))$, $\lambda^{\prime}\left(h_{\tau}(t)\right)=\lambda(t)$.

We consider a family $\mathcal{H}$ of surjective homomorphisms that has the following properties:

- it contains all identities,

- it is closed under composition,

- it has the diamond property: if $h_{1}: \mathcal{A} \rightarrow \mathcal{A}_{1}$ and $h_{2}: \mathcal{A} \rightarrow \mathcal{A}_{2}$ are in $\mathcal{H}$, there exist $h_{1}^{\prime}: \mathcal{A}_{1} \rightarrow \mathcal{A}^{\prime}$ and $h_{2}^{\prime}: \mathcal{A}_{2} \rightarrow \mathcal{A}^{\prime}$ in $\mathcal{H}$ such that $h_{1}^{\prime} \circ h_{1}=h_{2}^{\prime} \circ h_{2}$.

Under these hypothesis the relation $\mathcal{A}_{1} \sim_{\mathcal{K}} \mathcal{A}_{2}$ defined by $\exists h_{1}, h_{2} \in \mathcal{H}$ : $h_{1}\left(\mathcal{A}_{1}\right)=h_{2}\left(\mathcal{A}_{2}\right)$ is an equivalence relation.

It follows from the definition that each equivalence class for $\sim \mathcal{H}$ contains a unique minimal transition system.

Example 5. Bisinaulation homomorphisms The following family of homomorphisms was introduced in [4], and in [11, 16, 17] (under the name of transition preserving homomorphisms), to characterize the bisimulation relation between two transition systems $([29,25,20]$.

A surjective homomorphism $h: \mathcal{A} \rightarrow \mathcal{A}^{\prime}$ is a bisimulation homomorphism if

$$
\forall s_{1} \in S, \forall t^{\prime}=h_{\sigma}\left(s_{1}\right) \mapsto a \rightarrow s_{2}^{\prime} \in T^{\prime}, \exists t=s_{1} \mapsto a \rightarrow s_{2}: h_{\sigma}\left(s_{2}\right)=s_{2}^{\prime} .
$$

It is easy to show that this family has the above properties and that $\mathcal{A} \sim \mathcal{H} \mathcal{A}^{\prime}$ iff there is a bisimulation between $\mathcal{A}$ and $\mathcal{A}^{\prime}$.

\subsection{Logical equivalences}

Two transition systems are said to be logically equivalent with respect to some logic $L$ if they satisfy the same set of properties expressible in this logic. Restricting ourself to the logics introduced in the previous section, formulas of which are ground terms built up from a set $\Omega$ of sorted operators, we give the following definition: two transition systems $\mathcal{A}$ and $\mathcal{A}^{\prime}$ are logically equivalent with respect to $L$, denoted by $\mathcal{A} \sim_{L} \mathcal{A}^{\prime}$, if $\forall F \in L, F_{\mathcal{A}} \neq \emptyset \Leftrightarrow F_{\mathcal{A}^{\prime}} \neq \emptyset$.

\subsubsection{Indistinguishability}

This notion of logical equivalence is tightly related to the notion of $L$-type. Given a transition system $\mathcal{A}$ and an object $r$ of $\mathcal{A}$ of sort $\rho$, the L-type of $r$ is the set 
of formulas $\left\{F \mid r \in F_{\mathcal{A}}\right\}$. Two objects of the same sort are are said to be $L$-indistinguishable if they have the same $L$-type.

Obviously, if two transition systems $\mathcal{A}$ and $\mathcal{A}^{\prime}$ have the same sets of $L$-types (i.e., for each object $r$ of $\mathcal{A}$ there exists an object $r^{\prime}$ of $\mathcal{A}^{\prime}$ of the same $L$-type as $r$ ) then $\mathcal{A} \sim L \mathcal{A}^{\prime}$.

Conversely, let us assume that the sorts of $\Omega$ are such that any transition system $\mathcal{A}$ has only finitely many objects of each sort (it is the case for the sorts "states" and "transitions", not for the sort "paths"), and that $\Omega$ contains negation for all of its sorts. In this case, if $\mathcal{A} \sim_{L} \mathcal{A}^{\prime}$ then $\mathcal{A}$ and $\mathcal{A}^{\prime}$ have the same set of $L$-types. Let us assume that there is an object $r$ of $\mathcal{A}$ such that no object $r^{\prime}$ of $\mathcal{A}^{\prime}$ has the same $L$-type. For every object $r^{\prime}$ of $\mathcal{A}^{\prime}$ there is a formula $F_{r^{\prime}}$ such that $r \in\left(F_{r^{\prime}}\right)_{\mathcal{A}}$ and $r^{\prime} \notin\left(F_{r^{\prime}}\right)_{\mathcal{A}^{\prime}}$. Let us consider the finite conjunction $F$ of these $F_{r^{\prime}}$. Obviously $r \in F_{\mathcal{A}}$ while $F_{\mathcal{A}^{\prime}}$ is empty, a contradiction.

Let us remark that, under the same hypothesis, the same kind of reasoning allows us to prove that each $L$-type of $\mathcal{A}$ contains a characteristic formula $F$ in the sense that an object $r$ has this $L$-type iff $r \in F_{\mathcal{A}}$.

Let us also mention that, under these hypothesis, extending a logic with operators defined as least or greatest fixpoints does not extend its indistinguishability power: if the sort of the term $t\left(X, X_{1}, \ldots, X_{n}\right)$ contains only $k$ objects in $\mathcal{A}$, then $\left(\mu X . t\left(X, X_{1}, \ldots, X_{n}\right)\right)_{\mathcal{A}}$ is equal to $\left(V_{i=0}^{k} t_{i}\right)_{\mathcal{A}}$ where $t_{0}=0, t_{i+1}=t\left(t_{i}, X_{1}, \ldots, X_{n}\right)$. Therefore, the logical equivalence associated with this extension is the same as the original one.

\subsubsection{Quotients under indistinguishability}

In any transition system $\mathcal{A}$, the indistinguishability class of an object $r$ is the set of all objects of the same sort and of the same $L$-type.

Let us consider a logic $L$ containing only the sorts $\sigma$ and $\tau$ (if a logic contains only the sort $\tau$ we can extend it by adding transition formulas in the form $F \sim \sim_{a} F^{\prime}$ interpreted in a transition system $\mathcal{A}$ as the set of all transitions $s \mapsto a \rightarrow s^{\prime}$ such that $s \in F_{\mathcal{A}}$ and $\left.s^{\prime} \in F_{\mathcal{A}}^{\prime}\right)$. Let us consider the mapping $h=\left(h_{\sigma}, h_{r}\right)$ which sends each state and each transition on its indistinguishability class. We say that $L$ is adequate if this mapping is always a transition system homomorphism. We say that it is fully adequate if, moreover, $r$ and $h(r)$ have always the same $L$-type. In this case, $h(\mathcal{A})$ is the unique minimal transition system logically equivalent to $\mathcal{A}$ with respect to $L$.

Unfortunately, it is not always the case that a logic is fully adequate. However, fortunately, Hennessy-Milner logic as well as Dicky logic are fully adequate.

\subsection{Saturating homomorphisms}

The well known Hennessy-Milner theorem [20] asserts that the structural equivalence defined by the bisimulation relation is the same as the logical equivalence associated with the Hennessy-Milner logic. 
Along the line suggested by the notion of quotient under indistinguishability we are going to investigate the relation between the notions of structural and logical equivalence.

The main tool for studying this relation is the notion of a saturating homomorphism. Let $\Omega$ be a set of sorted operators containing only the sorts $\sigma$ and $\tau$. Let $\mathcal{A}$ and $\mathcal{A}^{\prime}$ be two transition systems. A surjective homomorphism

$h=\left(h_{\sigma}, h_{\tau}\right): \mathcal{A} \rightarrow \mathcal{A}^{\prime}$ saturates $\Omega$ if for all $\omega \in \Omega$ of sort $\rho_{1} \cdots \rho_{n} \rightarrow \rho$ and for all $P_{i}^{\prime} \subseteq E_{\rho_{i}}^{\prime}$

$$
h_{\rho}^{-1}\left(\omega_{\mathcal{A}}^{\prime}\left(P_{1}^{\prime}, \ldots, P_{n}^{\prime}\right)\right)=\omega_{\mathcal{A}}\left(h_{\rho_{1}}^{-1}\left(P_{1}^{\prime}\right), \ldots, h_{\rho_{n}}^{-1}\left(P_{n}^{\prime}\right)\right) .
$$

It is obvious that a surjective homomorphism always saturates the logical operators. The only interesting point is the saturation of the other operators.

Example 6. For instance, it is easy to see that a homomorphism is a bisimulation homomorphism iff it saturates the operators $\langle a\rangle$, for all $a \in A$.

For transition systems with unobservable transitions, labeled by $\epsilon$, there is a notion of branching bisimulation (cf. $[18,10])$. In exactly the same way as bisimulation is related to bisimulation homomorphisms, branching bisimulation is related to those homomorphisms that saturates the operator $\operatorname{src}_{\varepsilon}\left(Y_{\tau}\right)$, of sort $\tau \rightarrow \sigma$, defined as the least fixpoint of $X_{\sigma}=\operatorname{src}\left(Y_{\tau}\right) \vee\langle\varepsilon\rangle X_{\sigma}$.

It is a straightforward consequence of the definition of saturation that if $h: \mathcal{A} \rightarrow \mathcal{A}^{\prime}$ saturates $\Omega$, then, for every formula $F$ of the logic $L_{\Omega}$ built over $\Omega$, $h^{-1}\left(F_{\mathcal{A}^{\prime}}\right)=F_{\mathcal{A}}$; thus, $\mathcal{A}$ and $\mathcal{A}^{\prime}$ are logically equivalent with respect to $L_{\Omega}$.

It can be shown that the family $\mathcal{S}_{\Omega}$ of all homomorphisms saturating $\Omega$ contains identities and is closed under composition. If it has also the diamond property, it defines a structural equivalence $\sim_{\mathcal{S}_{\Omega}}$ which is included in the logical equivalence $\sim L_{n}$.

We have previously outlined the notion of a fully adequate logic. Indeed these logics have the nice following properties

- the canonical homomorphism from a transition system $\mathcal{A}$ onto its quotient under indistinguishability saturates $\Omega$,

from which we derive

- the family $\mathcal{S}_{\Omega}$ has the diamond property.

Therefore, for fully adequate logics, the structural equivalence $\sim \mathcal{S}_{\Omega}$ and the logical equivalence $\sim_{L_{\Omega}}$ are equal. 


\section{References}

[1] H. R. Andersen. Model checking and boolean graphs. In B. Krieg-Brückner, editor, ESOP '92, pages 1-19, Rennes, 1992. Lect. Notes Comput. Sci. 582.

[2] A. Arnold. Transition systems and concurrent processes. In Mathematical problems in Computation theory. Banach Center Publications,vol. 21, 1987.

[3] A. Arnold and P. Crubillé. A linear algorithm to solve fixed point equations on transition systems. Inf. Process. Lett., 29:57-66, 1988.

[4] A. Arnold and A. Dicky. An algebraic characterization of transition system equivalences. Information and Computation, 82:198-229, 1989.

[5] A. Arnold and M. Nivat. Comportements de processus. In Colloque AFCET "Les Mathématiques de l'Informatique", pages 35-68, 1982.

[6] G. Boudol. Notes on algebraic calculi of processes. In Logic and Models of Concurrent Systems, pages 261-303. NATO ASI Series F-13, Springer Verlag, 1985.

[7] G. W. Brams. Réseaux de Petri: théorie et pratique. Masson, Paris, 1982.

[8] E. M. Clarke, E. A. Emerson, and A. P. Sistla. Automatic verification of finite state concurrent systems using temporal logics specifications. ACM Trans. Prog. Lang. Syst., 8:244-263, 1986.

[9] R. Cleaveland and B. Steffen. Computing behavioural relations, logically. In 18th Int. Coll. on Automata, Languages and Programming, pages 127-138. Lect. Notes Comput. Sci. 510, 1991.

[10] R. de Nicola and F. Vaandrager. Three logics for branching bisimulation. In 5th Symp. on Logic in Comput. Sci., pages 118-129, 1990.

[11] P. Degano, R. D. Nicola, and U. Montanari. A partial ordering semantics for CCS based on condition/event systems. Acta Informatica, 26:59-91, 1988.

[12] A. Dicky. An algebraic and algorithmic method for analysing transition systems. Theoretical Comput. Sci., 46:285-303, 1986.

[13] E. A. Emerson. Temporal and modal logic. In J. Van Leeuwen, editor, Handbook of Theoretical Computer Science (vol. B), pages 995-1072. Elsevier, 1990.

[14] E. A. Emerson and J. Y. Halpern. "Sometimes" and "Not Never" revisited : on branching versus linear time temporal logic. J. Assoc. Comput. Mach., 33:151$178,1986$.

[15] E. A. Emerson and A. P. Sistla. Deciding full branching time logic. Information and Control, 61:175-201, 1984.

[16] G. L. Ferrari. Unifying models of concurrency. $\mathrm{PhD}$ thesis, Università di Pisa, 1990.

[17] G. L. Ferrari and U. Montanari. Towards the unification of models for concurrency. In A. Arnold, editor, CAAP '90, pages 162-176. Lect. Notes Comput. Sci. $431,1990$. 
[18] J. F. Groote and F. W. Vaandrager. An efficient algorithm for branching bisimulation and stuttering equivalence. Technical Report CS-R9001, Centre for Math. and Comput. Sci., Amsterdam, 1990.

[19] M. Hennessy. Algebraic theory of processes. The MIT Press, 1988.

[20] M. Hennessy and R.Milner. Algebraic laws for nondeterminism and concurrency. J. Assoc. Comput. Mach., 32:137-161, 1985.

[21] C. A. R. Hoare. Communicating sequential processes. Commun. ACM, 21:666$677,1978$.

[22] I. L. Ianov. The logical schemes of algorithms. In Problems of Cybernetics, volume 1, pages 75-127. Pergamon Press, 1960.

[23] R. M. Karp and R. E. Miller. Parallel program schemata. J. Comput. Syst. Sci., 3:147-195, 1969.

[24] R. M. Keller. Formal verification of parallel programs. Commun. ACM, 19:371$384,1976$.

[25] R. Milner. A calculus of communicating systems. Lect. Notes Comput. Sci. 92, 1980.

[26] R. Milner. Communication and concurrency. Prentice-Hall, 1989.

[27] M. L. Minsky. Computation: Finite and infinite machines. Prentice-Hall, 1967.

[28] M. Nivat. Sur la synchronisation des processus. Revue Technique ThomsonCSF, 11:899-919, 1979.

[29] D. Park. Concurrency and automata on infinite sequences. In 5th GI Conf. on Theoret. Comput. Sci., pages 167-183, Karlsruhe, 1981. Lect. Notes Comput. Sci. 104.

[30] J. D. Rutledge. On Ianov's program schemes. J. Assoc. Comput. Mach, 11:1-9, 1964.

[31] B. Vergauwen and J. Lewi. A linear algorithm for solving fixed point equations on transition systems. In J. Raoult, editor, CAAP '92, pages 321-341, Rennes, 1992. Lect. Notes Comput. Sci. 581.

[32] W. Zielonka. Notes on asynchronous automata. RAIRO Inform. Théor., 21:99$135,1987$. 